\title{
Semi-quantitative analysis of serum proteome in patients with bipolar disorder
}

\author{
Seregin Alexander Alexandrovich \\ Mental Health Research Institute \\ Tomsk National Research Medical \\ Center of the Russian Academy of \\ Sciences \\ Tomsk, Russia \\ email: apocalips1991@mail.ru
}

\author{
Smirnova Lyudmila Pavlovna \\ Mental Health Research Institute \\ Tomsk National Research Medical \\ Center of the Russian Academy of \\ Sciences \\ Tomsk, Russia \\ email:1psmirnova@yandex.ru
}

\author{
Simutkin German Gennadievich \\ Mental Health Research Institute \\ Tomsk National Research Medical \\ Center of the Russian Academy of \\ Sciences \\ Tomsk, Russia \\ email: ggsimutkin@gmail.com
}

\begin{abstract}
The work analyzed the protein spectrum of blood serum of 45 with bipolar disorder. Protein recruitment in BD was mainly associated with the immune response, regulation of transport processes through the cell membrane and cellular communication, the development of neurons and oligodendrocytes, and cell growth. Cadherin-5 and vascular endothelial growth factor receptor 1 are a marker of endothelial dysfunction. The detection of these proteins suggests the presence of endothelial dysfunction in the pathogenesis of $\mathrm{BD}$. The detection of myelin 1 transcription factor and the literature data on the effect of mutations in the MYT1L gene on the risk of major depressive disorder suggest the MYT1 protein as a potential BD biomarker. The results may help to discover new pathways associated $\mathrm{BD}$.
\end{abstract}

Keywords - biomarker, bipolar disorder, schizophrenia, proteome, mass spectrometry, serum

\section{Introduction}

Bipolar disorder (BD) leads to a significant decrease in the quality of life of patients and increases the risk of suicidal behavior. The disease often has a similar clinical picture with other mental disorders, and there are no paraclinical criteria for differential diagnosis for these disorders [1][2]. This is due to a lack of understanding of the molecular mechanisms of BD pathogenesis. In recent years, interest in proteomic research in mental disorders has increased and research in this direction has been intensively conducted [2][4][5]. Identification of the marker protein or regulatory proteins involved in the pathogenesis of BD in biomaterial available for diagnostic purposes - blood serum - will bring us as close as possible to understanding the specific pathogenetic mechanisms of this disorder and can serve as the basis for new methods of differential diagnosis and the development of new pathogenetically based medicines.

\section{Methods}

In this work, the protein spectrum of the blood serum of the following patient groups was analyzed 45 patients (aged $32[21,52]$ years and a disease duration of $8[5,11]$ years) with bipolar disorder (F31), 12 of them were in-patients of the Department of Affective States of MHRI and 11 were inpatients of the Department of Endogenous Mental Disorders and Affective States of the Mental Health Research Center (MHRC, Moscow). Patients were hospitalized in the acute state and blood serum samples were taken before the start of the study before the start of the course of therapy. The control group consisted of 24 mentally and somatically healthy volunteers matched to the study groups by gender and age. Exclusion criteria for all subjects were the presence of acute and chronic diseases in the acute phase; taking medication psychoactive drugs. Blood serum was taken from the ulnar vein in the morning on an empty stomach before the start of therapy. Further, the serum was purified by affinity chromatography from 6 major proteins (albumin, immunoglobulin G, immunoglobulin A, antitrypsin, transferrin and haptoglobin) on an AKTA pure chromatograph (GE Healthcare). After that, the purified proteins were concentrated to $1 \mathrm{ml}$ using Amicon Ultra-0.5 ultrafilters (MILLIPORE) at $3 \mathrm{kDa}$. and separated by vertical electrophoresis in a $12 \%$ polyacrylamide gel according to the Lemmli method (Laemmli, U.K, 1970). Then, after trypsinolysis and peptide extraction from the gel, the proteins were identified by HPLC / mass spectrometry on an LTQ Orbitrap Velos mass spectrometer (Thermo Scientific). was carried out in IBMC, Moscow (Centre of Collective Usage "Human proteome"). The mass spectrometric data were analyzed with the MaxQuant software (version 1.6.3.4). Default parameters were used unless otherwise specified below. A false discovery rate (FDR) of 0.01 for proteins and peptides and a minimum peptide length of 6 amino acids were required. The Andromeda search engine was used to search the MS/MS spectra against the UniProt human database combined with 262 common contaminants and concatenated with the reversed versions of all sequences. Enzyme specificity was set to trypsin specificity. A maximum of two missed cleavages were allowed. Peptide identification was based on a search with an initial mass deviation of the precursor ion of up to $7 \mathrm{ppm}$. The fragment mass tolerance was set to $20 \mathrm{ppm}$ on the $\mathrm{m} / \mathrm{z}$ scale. Only proteins quantified with at least two peptides were considered for quantitation. MS1-intensity based label-free quantitation was used to assess differences in the abundance of proteins between all the studied groups. LFQ intensities for the proteins were $\log 2-$ transformed and normalized to ensure equal median protein abundance across the samples. A two-tailed unpaired t-test with an FDR value of 0.05 and $\mathrm{S} 0=2$ was applied to identify proteins for which the abundance was significantly changed between all the studied groups.

\section{Results}

As a result of mass spectrometric analysis, between 50 and 350 proteins in each lane were identified and about 1,600 proteins were identified for each person. Comparison of the proteome profiles of different groups revealed 18 proteins specific for BD.

Proteins found in patients with $\mathrm{BD}$, but missing from the control sample, are involved in the regulation of DNA and cell 
cycle synthesis, differentiation of progenitor cells, in the development of neurons and oligodendrocytes, such as Myelin transcription factor 1 (MYT1; iBAQ 607,717; LFQ $8,153,738$ ), which regulates genes encoding myelin proteins and other central nervous system proteins [6]. This protein is a zinc finger DNA-binding protein that affects the proliferation, differentiation and transcription of myelin cell genes. MYT1 can regulate the critical transition point in oligodendrocyte lineage development by modulating oligodendrocyte progenitor proliferation relative to terminal differentiation and up-regulation of myelin gene transcription [7]. Ti Wang et al. Have shown that mutations in the MYT1L gene encoding a protein of the myelin transcription factor 1 family can increase the risks of major depressive disorder. However, a connection with pathogenesis has not yet been identified [8]. A significant increase in the expression of the MYT1 gene in the prefrontal cortex of patients with BD is also known. These facts suggest the MYT1 protein as a potential biomarker for BD. Desmoplakin (DSP iBAQ 3,736,801; LFQ $5,864,175)$ was also found in the serum of patients with BD, which participates in the organization of cadherin-placoglobin desmosome complexes, forming them in separate domains of the plasma membrane. Cadherin 5 (CADH5 iBAQ 4,472,995; LFQ $6,134,045)$ was also found in a group of patients with BD. The cadherin protein family not only provides mechanical contact between neighboring cells, but also participates in intracellular signal transduction, regulation of proliferation, migration, sorting, differentiation, and cell morphogenesis. In the tissues of adult organisms, cadherins regulate cell renewal, provide a physiological barrier between contacting tissues and selectivity of the transport of soluble substances. Some mediators of the inflammatory response, such as the vascular endothelial growth factor (VEGFR1) (FLT1; iBAQ 4,665,292; LFQ 6,331,822) we discovered, can interfere with the organization of contacts when their receptors are bound, thereby opening the barrier, and plasma proteins can pass through the endothelial barrier. In particular, VEGFR1 is involved in the initiation of autophosphorylation of cadherin 5 signaling cascades, which directly affect the development of endothelial dysfunction [9]. In addition, it is known that cadherins mediate cell sorting, migration, and segregation, morphogenesis, and axon growth in embryogenesis [10]. Van et al. The involvement of genes encoding the CDH9 and CDH10 families have been demonstrated in autism [11]. However, there is no data yet, which indicates the participation of cadherin 5 in the pathogenesis of mental disorders, which requires further study.

\section{Conclusions}

Comparison of the proteome profiles of the studied groups revealed 18 proteins specific for BD. The set of proteins in BD was mainly associated with the immune response, regulation of transport processes across the cell membrane, and cell communication. Many of these pathways are involved in the pathogenesis of mental disorders. They are interesting as potential markers. Cadherin-5 and vascular endothelial growth factor receptor 1 are a marker of endothelial dysfunction. The detection of these proteins suggests the presence of endothelial dysfunction in the pathogenesis of BD. Detection of desmoplakin can indirectly indicate a violation of the intercellular contacts of endotheliocytes. The detection of myelin 1 transcription factor and the literature data on the effect of mutations in the MYT1L gene on the risk of major depressive disorder suggest the MYT1 protein as a potential BD biomarker. However, its role in the pathogenesis of BD remains to be seen. Further study of the proteins that we identified that claim to be BD biomarkers may help to uncover unclear aspects of pathogenesis and develop new paraclinical criteria for the differential diagnosis of BD.

\section{ACKNOWLEDGMENT}

The study was carried out as part of the government assignment (budgetary funding within the framework of the comprehensive research topic AAAA-A19-119020690013-2 "A comprehensive study of clinical-psychopathological patterns and pathobiological mechanisms of the formation and progression of socially significant mental and behavioral disorders with the development of innovative methods of early diagnosis, personalized treatment and prevention strategies"). Mass spectrometric experiments were performed the equipment of "Human Proteome" Core Facility of the Institute of Biomedical Chemistry (Moscow).

We thank Dr Alexey Chernobrovkin, PhD, Department of Bioinformatics, the Institute of Biomedical Chemistry (IBMC) Moscow, Russia, for his help in work with the MaxQuant software

\section{REFERENCES}

[1] Kornetov A. N. " Ontogenetic aspects of depressive disorders," vol. 103(8), S.S. Korsakov Journal of Neurology and Psychiatry. 2003, pp. 80-81.

[2] Marusin A.V., Kornetov A.N. and Swarovskaya M.G. "Association of genes for susceptibility to alcoholism, schizophrenia and Alzheimer's disease with psychodiagnostic traits in the Russian population," vol. 15(5), Bulletin of Siberian Medicine. 2016, pp. 83-96. doi: 10.20538/1682-0363-2016-5-83-96

[3] Smirnova L.P., Loginova L.V., Dmitrieva E.M., Seregin A.A., Semke A.V., Simutkin G.G., Ivanova S.A. "The first results of comparison of blood serum proteomes of patients with schizophrenia and bipolar affective disorder," vol. 2 (91), Siberian Herald of Psychiatry and Addiction Psychiatry. 2016, pp. 42-47.

[4] Dudley E., Hassler F. and Thome J. "Profiling for novel proteomics biomarkers in neurodevelopmental disorders," vol. 8(1) Expert Rev Proteomics. 2011, pp. 127-36. doi: 10.1586/epr.10.97.

[5] Föcking M., Dicker P. and English J.A. "Common proteomic changes in the hippocampus in schizophrenia and bipolar disorder and particular evidence for involvement of cornu ammonis regions 2 and 3," vol. 68(5), Arch Gen Psychiatry. 2011, pp. 477-88. doi: 10.1001/archgenpsychiatry.2011.43.

[6] Singh S. M., Castellani C. and O'Reilly R. "Autism meets schizophrenia via cadherin pathway," vol. 116, Schizophr Res. 2010, pp. 293-4.

[7] Ryan, M., Lockstone, H. and Huffaker. "Gene expression analysis of bipolar disorder reveals downregulation of the ubiquitin cycle and alterations in synaptic genes," vol. 11, Mol Psychiatry. 2006, pp. 965978.

[8] Ti Wang, Zhen Zeng and Tao Li. "Common SNPs in Myelin Transcription Factor 1-Like (MYT1L): Association with Major Depressive Disorder in the Chinese Han Population," vol. 5(10), Yongyong Shi PLoS One. 2010, doi: 10.1371/journal.pone.0013662

[9] Pang V, Bates DO and Leach L. "Regulation of human feto-placental endothelial barrier integrity by vascular endothelial growth factors: competitive interplay between VEGF-A165a, VEGF-A165b, PIGF and VE-cadherin," vol. 23;131(23), Clin Sci (Lond). 2017

[10] Perez-Moreno M, Jamora C and Fuchs E. "Sticky business: orchestrating cellular signals at adherens junctions," vol. 112(4), Cell. 2003, pp. 535-548.

[11] Wang K, Zhang H and Ma D, "Common genetic variants on 5p14.1 associate with autism spectrum disorders," vol. 28;459(7246), Nature. 2009 , pp. 528-533. 